\title{
The histology of peau d'orange in breast cancer - what are the implications for surgery?
}

\author{
W S Wiggett, MB ChB, MMed (Surg) \\ Department of Surgery, University of Pretoria and Steve Biko Academic Hospital, Pretoria \\ M Louw, MB ChB, MMed (Anat Path) \\ Department of Anatomical Pathology, University of Pretoria, and National Health Laboratory Service, Tshwane Academic \\ Division
}

V O L Karusseit, MB ChB, MFGP (SA), MMed (Surg), FCS (SA)

Department of Surgery, University of Pretoria and Steve Biko Academic Hospital, Pretoria

Introduction. Surgery is sometimes performed on patients with peau d'orange (dermal oedema) of the breast. This may be done to achieve local control of cancer after neo-adjuvant chemotherapy or in resectable locally advanced disease. Conventional practice is not to place excision lines through areas of peau dorange for fear of recurrence in such an area. The question can be asked whether this wisdom is still valid in modern practice. No formal cohort studies documenting the histopathology of the skin in areas of peau dorange have been published, and available descriptions are scanty. Aim. To describe the histopathological features of peau d'orange. Method. Consecutive patients undergoing mastectomy for cancer in whom peau d'orange was present were selected over a period of 2 years. Blocks of skin were excised from areas of peau d'orange and examined histologically. The presence, nature and location of malignant cells were recorded and correlated with lymph node pathology. Prior administration of neo-adjuvant chemotherapy was noted.
Results. Twenty-six mastectomy specimens were examined. Tumour islands in lymphatics were identified in 10 of the 26 specimens. These tumour groups were found in lymph vessels of both the superficial and deep dermal plexuses. In 1 specimen the presence of malignant cells was equivocal. Metastatic tumour was present in axillary lymph nodes in 19 of 22 specimens. Fourteen patients had been treated with neo-adjuvant chemotherapy, and 5 of their specimens exhibited the presence of tumour cell groups in lymphovascular channels.

Conclusion. Tumour cells were present in the lymphatic vessels in areas of peau d'orange in $38 \%$ of the specimens studied. It would be expected that placing an excision line in such an area would result in an incomplete cancer operation in a high percentage of, but not all, cases.

S Afr J Surg 2012;50(3):75-78. DOI:10.7196/SAJS.1103 
Haagensen considered clinical criteria of operability in breast cancer. From a series of 1544 patients he concluded that patients with extensive oedema of the skin over the breast were doomed. None was disease-free 5 years after radical surgery. ${ }^{1}$ Haagensen also categorised limited oedema of the skin in breast cancer as a 'grave sign'. Five-year survival was reduced by half in patients with this sign.

Peau d'orange in breast cancer is thought to be due to occlusion of lymphatics by tumour emboli. The microscopic appearance of oedematous skin was described by Haagensen. ${ }^{2}$ He found dermis thickened by oedema and occasional emboli of carcinoma cells in dilated deep dermal lymphatics. This appearance was seen in superficial dermal lymphatics in cases with severe pitting oedema.

In the era before Haagensen, surgeons advocated radical local excision of breast cancer including clear margins around all areas of peau d'orange. This theory of breast cancer as a localised disease of the breast to be treated by surgery was later replaced by one of breast cancer being a systemic disease early in its course. Currently breast cancer is treated by multiple modalities. ${ }^{3}$ This is especially the case in locally advanced disease, including all cases exhibiting dermal oedema (T4b and T4d).

Contemporary practice still precludes placing surgical resection lines through areas of peau d'orange. The question can be asked whether this wisdom is still valid in modern practice. This is especially apposite to the current practice of neo-adjuvant therapy and breast conservation surgery.

No formal cohort studies of the histopathology of the skin in areas of peau d'orange have been published. Available descriptions are scanty or are part of studies reporting other aspects of breast cancer. ${ }^{2,4,5}$

\section{Aim}

The aim of this study was to describe the histopathology of peau d'orange and to determine the presence and location of the lymphovascular invasion (LVI) in the skin of the breast in areas of oedema.

\section{Material and methods}

A prospective study of specimens submitted for histological examination from patients with proven breast cancer was undertaken over a period of 2 years. Mastectomy specimens that exhibited peau d'orange were selected. Peau d'orange was defined in this study as any clinically apparent area of oedema of the skin of the breast. The mastectomies had been performed primarily, or secondarily after neo-adjuvant chemotherapy, in cases of breast cancer in which the areas of peau d' orange could be safely encompassed by surgical excision. All the patients presented with locally advanced breast cancer (T4 lesions), and only 2 had inflammatory cancer (T4d).

The breast tissue and lymph nodes were routinely processed to formalin-fixed paraffin wax-embedded blocks and stained with haematoxylin and eosin. Several additional blocks, depending on the size of the affected area, from skin in the areas of peau d'orange were also processed. These were taken well away from any tumour and examined for the presence of tumour cells in the dermal lymphatics.
LVI was defined as the presence of groups of tumour cells in vascular channels lined by endothelial cells without supporting smooth muscle. Care was taken to exclude cases exhibiting shrinkage artefact, which could give a false impression of lymphovascular invasion. ${ }^{6}$ Areas adjacent to the primary tumour were avoided to prevent the inclusion of tissue containing local direct infiltration of lymphatics and to preclude examining tissue in which local effects of the tumour could cause oedema. ${ }^{6}$

LVI was correlated with lymph node metastases in each individual case. Prior treatment with neo-adjuvant chemotherapy was also noted.

\section{Results}

Twenty-six mastectomy specimens were examined. Axillary dissection had been performed in 22 patients and simple mastectomy in 4. Histological examination showed infiltrating ductal carcinoma in 24 of the 26 specimens and metaplastic carcinoma in 2.

Tumour groups were identified in sections of skin in areas of peau d'orange well away from the invasive tumour in 10 of the 26 mastectomy specimens (38\%). These tumour groups were found in lymphatic spaces of both the subepithelial and subdermal plexuses in all specimens. In 1 specimen the presence of tumour cell groups in the lymphovascular spaces was equivocal. The location of the subepithelial or dermal lymphatic invasion varied from case to case. In some cases the location was predominantly in the subepithelial lymphatic channels (Fig. 1), while in others the location was predominantly in the deeper subdermal lymphatic plexus (Fig. 2). In all cases the tumour cells were present within the lymphovascular spaces as groups with no apparent attachment to the endothelial lining.

In most of the sections of the areas that did not exhibit LVI the lymphatic channels appeared dilated. In some of these cases limited oedema of the adjacent tissue was present. No malignant cells were present outside lymphatic channels in any of the sections examined.

Metastatic tumour was present in axillary lymph nodes in 19 of 22 specimens. Table 1 shows the relationship of LVI to the presence of lymph node metastases and prior administration of chemotherapy

Eight of the 10 specimens in which LVI could be identified exhibited metastatic tumour in the lymph nodes examined from the axillary dissection. The remaining patients had not undergone axillary dissection.

LVI was absent in the skin sections of 15 of the 26 specimens examined. Eleven of these 15 cases had metastatic tumour in the axillary lymph nodes. Only 1 patient in this group had not undergone an axillary dissection.

Fourteen of the patients had received neo-adjuvant chemotherapy. LVI could be identified in the skin lymphatics in 5 of these patients.

In the 12 patients who did not receive neo-adjuvant chemotherapy, LVI in the skin was present in 5 patients, absent in 6 and equivocal in 1 .

\section{Discussion}

Apart from cancer, there are many causes of oedema of the breast such as occlusion of the subclavian vein, mastitis, congestive cardiac failure, and axillary lymph node disease such as 


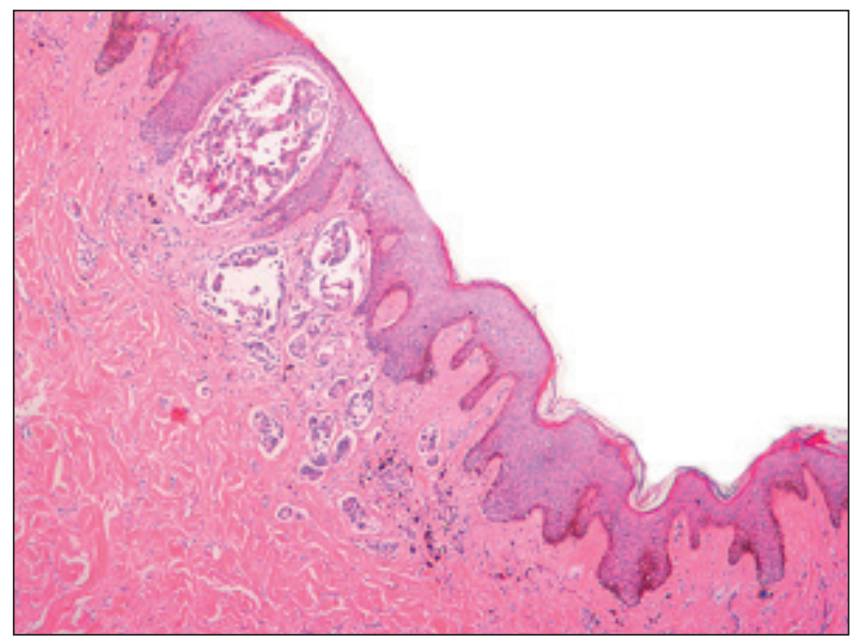

Fig. 1. Lymphovascular invasion in the superficial plexus of the skin.

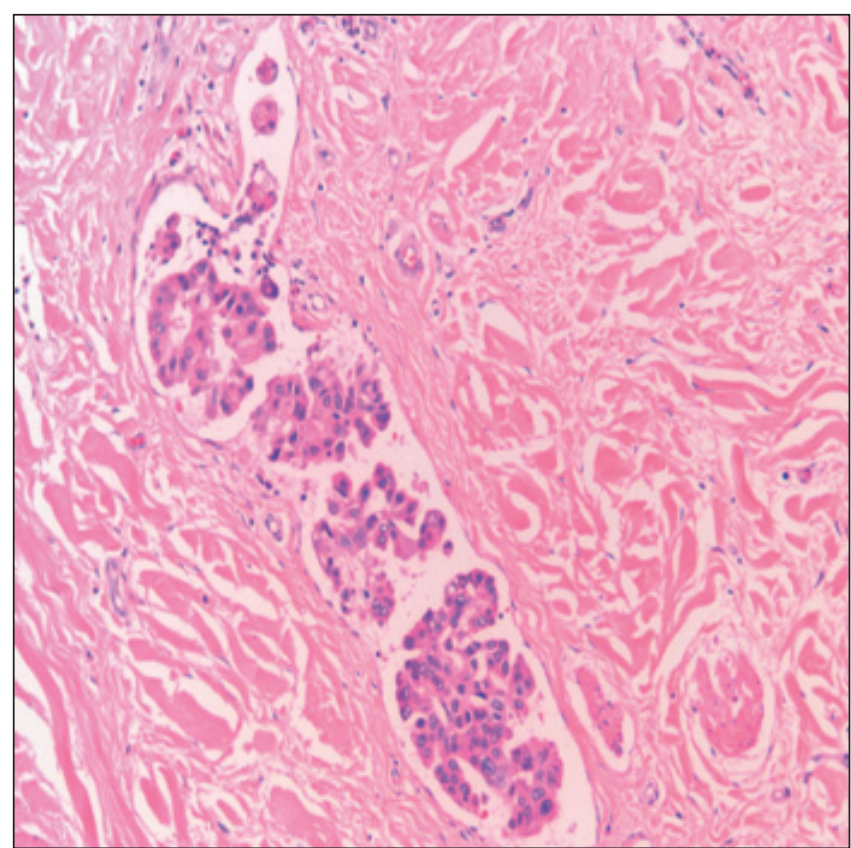

Fig. 2. Lymphovascular invasion in the deep dermis.

tuberculosis. ${ }^{7}$ None of these were present in the cases in this study. All patients had proven breast cancer, and oedema of the skin was assumed to be due to malignant lymphatic invasion.

The aim of this study was simply to describe the histological appearance of the skin exhibiting peau d'orange in breast cancer. No special staining was performed and no features sought that have been described in breast tissue related to the behaviour of breast cancer or its response to therapy. These aspects have been reported in several studies. ${ }^{5,8-10}$
Handley described the lymphatic systems in the skin of the breast. ${ }^{11}$ Dermal papillary and subpapillary channels constitute the subepithelial plexus. This plexus is valveless and is connected to the valved deep dermal plexus by vertical channels. These deep lymphatics drain unidirectionally to regional lymph nodes. ${ }^{12}$ This dermal lymphatic system was the objective of this study.

Tumour cell groups were identified in lymphatics in 10 of 26 specimens. These occurred in lymphatic channels in both the subepidermal and the subdermal plexuses. These deposits had the appearance of emboli in that they occurred as clusters of cells. They also appeared not to be attached to endothelium. This appearance is similar to that described in literature as tumour embolisation. ${ }^{6,13}$ This might be the explanation for the absence of malignant cells in the lymphatics of some specimens, in that the random sections might have cut through lymphatics containing no emboli.

Metastatic tumour was present in the axillary lymph nodes in 11 of 15 breasts with no lymphovascular invasion. This could further support the embolic theory as described in the literature..$^{14}$ If tumour groups enter the lymphovascular drainage system as emboli, rather than growing as continuous strands in the lymphatic system, malignant cells may not be visible in all sections examined. It is furthermore striking that the vast majority of the patients with peau d'orange exhibited lymph node metastases: 8 of 10 with, and 11 of 15 without, demonstrable lymphovascular invasion in the skin.

Neo-adjuvant chemotherapy had been administered to 14 patients. Five of these patients' specimens exhibited LVI in the skin, compared with 5 patients in the group of 12 that had not received neo-adjuvant chemotherapy. This is a similar proportion, although it was not the aim of this study to investigate the response of LVI to chemotherapy. It has, however, been hypothesised that tumour in lymphatic vessels is relatively non-responsive to adjuvant therapy. ${ }^{15,16}$

Reports on tumour embolisation in breast cancer are problematic because tumour embolisation is not always defined. The definition is also not clear in the literature. ${ }^{8,9}$ The definition used here is that of a group of malignant cells within an endothelial-lined space lacking a supporting smooth-muscle layer. Rosen, in addition, prescribes the absence of red blood cells in such spaces. ${ }^{6}$

An additional problem is inter- and intra-observer variation in identification of lymphatic tumour emboli. This is well-known phenomenon in breast as well as other cancers. ${ }^{6,17}$ In 35 specimens of breast cancer reported by Gilchrist et al. ${ }^{18}$ complete agreement between pathologists regarding intra-mammary lymphatics was reached in only 12 cases. In 5 of the 35 cases there was complete disagreement and in 18 cases there was partial agreement. Other

Table 1. Lymphovascular invasion by category in the skin in areas of dermal oedema in mastectomy specimens $(N=26)^{*}$

\begin{tabular}{|c|c|c|c|c|}
\hline & LN metastases present & LN metastases absent & Neo-adjuvant therapy & No neo-adjuvant therapy \\
\hline LVI present $(n=10)$ & 8 & 2 & 5 & 5 \\
\hline LVI absent $(n=15)^{\dagger}$ & 11 & 3 & 9 & 6 \\
\hline${ }^{*}$ LVI uncertain in 1 specimen. & \multicolumn{3}{|c|}{${ }^{\dagger}$ One patient did not undergo LN dissection. } & \\
\hline LN = lymph node & & & & \\
\hline
\end{tabular}


similar studies also investigated intra-mammary and not dermal lymphatics. ${ }^{8,9,19}$

We are satisfied that this description of the appearance of lymphatics in skin exhibiting peau d'orange in this study accurately reflects the presence of LVI of the skin. It would be expected that the presence of malignant cells in lymphatic spaces in the skin well away from the primary tumour be more accurately diagnosed than in intra-mammary lymphatics in the breast parenchyma. All the sections were of skin only and were examined microscopically by a single pathologist. All the cases complied with the above definition of tumour embolisation, except for 1 in which this was uncertain.

Placement of excision lines across areas of peau dorange would violate surgical oncological principles of 'not cutting through cancer' in a substantial proportion of cases. Current practice requires that radiotherapy to the chest wall be administered after surgery for locally advanced cancer of the breast, ${ }^{20}$ but it would still seem to be prudent not to leave areas of peau d'orange behind by cutting through such affected skin.

Neo-adjuvant chemotherapy may possibly play a role in clearing lymphatics of malignant cells. There was no difference in the occurrence of lymphovascular infiltration between groups that had and had not received chemotherapy in this study. None of the several published systems for evaluating pathological complete response after chemotherapy includes investigation of skin lymphatics. ${ }^{9}$ A formal study investigating this aspect needs to be conducted.

\section{Conclusion}

This was a descriptive study only. No statistical conclusions can be drawn. Nevertheless, in a significant number of mastectomy specimens, malignant cells were identified in lymphatics of the skin in areas of peau d'orange. It would therefore be expected that placing an incision line in such an area would result in an incomplete cancer operation and possible local recurrence in a high percentage of, but not all, cases (38\%). We therefore recommend that the conventional practice of not placing incisions through areas of peau d'orange be maintained.
REFERENCES

1. Haagensen C, Stout A. Carcinoma of the breast: criteria for operability. Ann Surg 1943;118:859-868.

2. Haagensen CD. Diseases of the Breast. 3rd ed. Philadelphia: WB Saunders, 1986

3. Shenkier T, Weir L, Levine M, Olivotro I, Whelan T, Reyno L. Clinical practice guidelines for the care and treatment of breast cancer: 15. Treatment for women with stage III or locally advanced breast cancer. CMAJ 2004;170(6):983-994

4. Ellis DL, Teitelbaum SL. Inflammatory carcinoma of the breast. Cancer 1974;33:10451047.

5. Gruber G, Ciriolo M, Altermatt HJ, Aebi S, Berclaz G, Greiner RH. Prognosis of dermal lymphatic invasion with or without clinical signs of inflammatory breast cancer. Int J Cancer 2004;109:144-148.

6. Rosen PP. Tumor emboli in intramammary lymphatics in breast carcinoma: pathologic criteria for diagnosis and clinical significance. Pathology Annual 1983;18(2):215-232.

7. Kwak JY, Kim E, Chung SY, et al. Unilateral breast edema: spectrum of etiologies and imaging appearances. Yonsei Med J 2005;46(1):1-7.

8. Horvath Z, Torday L, Hitre E, et al. Inflammatory breast cancer - comparing the effectivity of preoperative docetaxel-epirubicine protocol to conventional anthracycline-containing chemotherapy to achieve clinical benefit and complete pathlogical response. Pathol Oncol Res 2011;17:541-550.

9. Mailliez A, Baranzelli MC, Giard S, et al. Is there a reliable method to assess the complete pathologic response on the tumor after neo-adjuvant chemotherapy in inflammatory breast cancer toward recommendations for the pathologic process? Experience in 56 patients treated in a single institution. Breast J 2010;16:464-471.

10. Sinn HP, Schmid H, Junkerman $H$, et al. Histologic regression of breast cancer after primary (neoadjuvant) chemotherapy. Geburtshilfe und Frauenheilkunde 1994;54(10):552-558.

11. Handley RS. The Anatomy of the Breast: Breast Cancer and its Diagnosis and Treatment. Baltimore: Williams \& Wilkins, 1955.

12. Kuhns JG, Ackerman DM. Macroscopic anatomy of the breast. In: Donegan WL, Spratt JS, eds. Cancer of the Breast. 5th ed. St Louis: Saunders, 2002.

13. Orbo A, Stalsberg H, Kunde D. Topographic criteria in the diagnosis of tumor emboli in intramammary lymphatics. Cancer 1990;66:972-977.

14. Nime FA, Rosen PP, Thaler HT, Ashikari R, Urban JA. Prognostic significance of tumor emboli in intramammary lymphatics in patients with mammary carcinoma. Am J Surg Pathol 1977;1(1):25-30.

15. Moore DH, Rouse MB, Massenburg GS, Zeman EM. Description of a spheroid model for the study of radiation and chemotherapy effects on hypoxic tumor cell populations. Gynecol Oncol 1992;47:44-47.

16. Alpaugh ML, Tomlinson JS, Ye Y, Barsky SH. Relationship of sialyl-Lewis ${ }^{\mathrm{x} / \mathrm{a}}$ underexpression and E-cadherin overexpression in the lymphovascular embolus of inflammatory breast cancer. Am J Pathol 2002;161(2):619-628.

17. Harris EL, Lewin DN, Wang HL, et al. Lymphovascular invasion in colo-rectal cancer. An interobserver variability study. Am J Surg Pathol 2008;32(12):1816-1821.

18. Gilchrist KW, Gould VE, Hirschl S, et al. Interobserver variation in the identification of breast carcinoma in intramammary lymphatics. Hum Pathol 1982;13(2)170-172.

19. Van den Eynden GG, Van der Auwera I, Van Laere SJ, et al. Distinguishing blood and lymph vessel invasion in breast cancer: a prospective immunohistochemical study. $\mathrm{Br}$ J Cancer 2006;94(11):1643-1649.

20. Dawood S, Merajver SD, Viens P, et al. International expert panel on inflammatory breast cancer: consensus statement for standardized diagnosis and treatment. Ann Oncol 2011;22(3):515-523. 